\title{
Influence of the High-Power Ion-Beam Irradiation of a Hydroxyapatite Target on the Properties of Formed Calcium Phosphate Coatings
}

\author{
G. A. Bleykher, T. L. Volokitina, and S. I. Tverdokhlebov \\ Tomsk Polytechnic University, Tomsk, Russia \\ Received February 1, 2013
}

\begin{abstract}
The physical-mechanical of properties of biocompatible calcium phosphate coatings deposited onto titanium and silicon substrates from erosion materials, which are generated by irradiating hydroxyapatite (synthetic and natural) targets by means of the high-power pulsed ion beam of a Temp-4 accelerator, are investigated. A calculation technique for predicting the rate and energy efficiency of deposition using pulsed ion beams is proposed. Their characteristics are analyzed as applied to the formation of calcium phosphate coatings.
\end{abstract}

\section{INTRODUCTION}

As was reliably proved by numerous investigations, high-power pulsed ion beams (HPPIBs) with particle energies of $100-1000 \mathrm{keV}$ and power densities of $10^{7}-$ $10^{9} \mathrm{~W} / \mathrm{cm}^{2}$ are capable of rapidly removing a substance from an irradiated surface and can serve as a tool for the deposition of modifying coatings with different compositions and purposes. The published experimental data confirm a number of attractive properties in technologies based on modern HPPIBs. In particular, they make it possible to retain the stoichiometric composition of the target material in deposited coatings and ensure their purity. In addition, a large treatment area (tens of square centimeters) and acceptable efficiency are achieved [1-3]. With the help of HPPIBs, a biocompatible calcium phosphate (CP) coating can be deposited onto the surfaces of different implants used in dentistry, traumatology, and orthopedics [4-6]. The base of these products is most often fabricated from titanium and its alloys or corrosionresistant high-quality steel, and $\mathrm{CP}$ coatings stimulate the osteogenesis of bone tissues and implant fixing due to the coalescence of the coating material and bone.

In the development of reliable and efficient HPPIB-based technologies intended for the creation of CP coatings, an important problem lies in reaching balance between the productivity and quality of the formed coatings. It is necessary to reveal HPPIB parameters at which the produced coatings not only can satisfy biomedical requirements but also ensure the highest indices of productivity and energy efficiency of basic technological processes.

Investigations into the properties of $\mathrm{CP}$ coatings deposited by means of submicron ion beams have demonstrated that they are capable of complying with requirements to medical products. It was confirmed that these coatings are untoxic and apyrogenic, do not give rise to degenerative changes in tissue during implantation, and meet the requirements of regulations. However, they are deposited at very low rates (no more than several nanometers within a single beamcurrent pulse) [4-7]. However, there is no explicitness in the question as to whether the accelerator operation modes used in experiments are optimal from the standpoint of the energy efficient obtainment of a deposited material.

The efficiency of beam-plasma coating deposition technologies is determined mainly by the intensity and energy efficiency of substance removal from the irradiated target surface. In connection with this, the use of HPPIBs has an advantage over many other known methods. For example, when the surface erosion of a solid body is caused by evaporation under the action of HPPIBs with the initial particle energy $E_{0}=10-$ $1000 \mathrm{keV}$ and power densities of $10^{7}-10^{9} \mathrm{~W} / \mathrm{cm}^{2}$, the erosion coefficients are three-four orders of magnitude higher than the coefficients of sputtering produced by low-current ion beams or magnetron discharge plasma $[8,9]$. The efficiency of submicrosecond ion accelerators is $20-40 \%$, substantially exceeding that of pulsed laser sources $[5,10]$. In addition, unlike laser radiation, ion beams with particle energies of more than $10 \mathrm{keV}$ are scarcely reflected from a target surface.

This study is aimed at analyzing the properties of $\mathrm{CP}$ coatings formed by erosion-material deposition from synthetic and natural hydroxyapatite (HAP) targets, which is implemented as a result of their irradiation by powerful pulsed ion beams of submicrosecond duration in a modern Temp-4M accelerator [10]. The productivity and energy efficiency of deposition are 
investigated with the help of numerical models describing the surface erosion of a solid body under exposure to high-power pulsed beams of charged particles $[8,9]$. Their application to the solution of the given problem enables us to predict the deposition efficiency and reveal the optimal parameters of irradiation.

\section{EXPERIMENTAL}

The schematic diagram of coating deposition by means of HPPIBs is presented in Fig. 1. High-energy ion-beam particles bombard a solid target surface at angle $\theta$ to its normal. Owing to their deceleration, particles (atoms, molecules, and others) are emitted from the target surface layers. Their flux is commonly called ablative or erosive. The flux propagates into the ambient space and is deposited on the substrate arranged parallel or at a small angle to the target surface at the distance $L$. The whole process occurs in high vacuum (the chamber pressure is $10^{-4}-10^{-2} \mathrm{~Pa}$ ).

In this study, two targets $\left(\mathrm{Ca}_{10}\left(\mathrm{PO}_{4}\right)_{6}(\mathrm{OH})_{2}\right)$, which were fabricated from HAP of different nature under different annealing conditions, are employed. One target was prepared by compressing a synthetic HAP powder obtained by means of the mechanochemical method according to ceramic technology [11] with the addition of silicon. The preparation was annealed in air at a temperature of $1100^{\circ} \mathrm{C}$. This target is hereinafter referred to as synthetic. The other target was fabricated from a natural HAP powder and is called natural. The obtained preparation was annealed in air at a temperature of $950^{\circ} \mathrm{C}$.

The submicrosecond ion beam generated in the Temp-4M accelerator, under the action of which an erosion flux from the target surface was created, had the following parameters [10]: an accelerating voltage of 200-250 keV; a beam-current pulse duration of approximately $80 \mathrm{~ns}$ (the current comprised $70 \%$ of $\mathrm{C}^{+}$ and $30 \%$ of $\mathrm{H}^{+}$); the current density on the target was $150-200 \mathrm{~A} / \mathrm{cm}^{2}$; the beam-trace radius on the target surface was about $2.5 \mathrm{~cm}$, and the angle of beam incidence on the target, $\theta$, was approximately $45^{\circ}$ (Fig. 1). The distance $L$ between the target and substrate surfaces was $6-7 \mathrm{~cm}$. Coatings were formed using a series of pulses with frequencies of $0.1-1 \mathrm{~Hz}$.

The erosion fluxes were deposited onto titanium and silicon substrates. The titanium substrate surfaces were preliminarily mechanically polished by means of a GOI paste. To improve the adhesive properties of the coating, the substrates were heated to $350^{\circ} \mathrm{C}$ during the experiments.

The elemental compositions of the coatings deposited onto titanium substrates were determined using a Shkhuna-2 Auger spectrometer by means of a Philips SEM 515 scanning electron microscope equipped with a built-in energy-dispersive X-ray analyzer. Energy-dispersive $\mathrm{X}$-ray analysis, also known as energy-dispersive spectroscopy (EDS), provides spectra that make it possible to estimate the proportions of

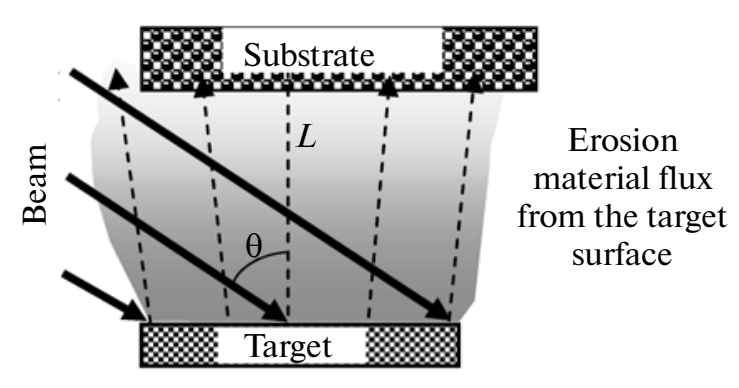

Fig. 1. Diagram illustrating the deposition of coatings from erosion material escaping from the surface layers of the target subjected to the action of HPPIBs.

different elements in the coating. The physicalmechanical properties of the deposited coatings were studied via the indentation method using a NanoScan beam profiler. The CP coating thicknesses were measured using a Talysurf 5 profilometer-profilograph with the use of polished silicon substrates. Toxicological tests were performed at the certified test laboratory center of the Novosibirsk Research Institute of Hygiene.

\section{EXPERIMENTAL RESULTS AND DISCUSSION}

The Auger spectra of the CP coatings, which were obtained using synthetic and natural targets subjected to the action of 300 HPPIB pulses, are depicted in Fig. 2. These coatings contain the basic components of a HAP target and titanium substrate: $\mathrm{Ca}, \mathrm{P}, \mathrm{O}$, and Ti. Foreign impurities and the base material (titanium) are not observed on the coating surfaces. At a certain depth below the surface, the coating material mixes with titanium. The Auger spectra enable us to infer that the coating fully covers the substrate, providing good adhesion to it. These properties are necessary to protect implants because, in this case, contact between the living tissue of an organism and the base material is eliminated. As is seen in Fig. 2, the coating deposited due to natural target erosion is thicker than that obtained with the use of a synthetic target by approximately an order of magnitude. It is precisely this circumstance that explains the difference in the depth of mixing between the coating and substrate elements.

The proportions of different elements in the coatings, which were determined via energy-dispersive analysis, are summarized in Table 1 . To reduce the influence of the substrate material on the spectra, an analysis was carried out at a reduced voltage of $10 \mathrm{kV}$. Before this was carried out, their surfaces were covered with conducting silicon layers $5 \mathrm{~nm}$ thick because the $\mathrm{CP}$ coatings are dielectric. Hence, the EDS spectra of the coatings contain silicon. For synthetic and natural targets, the $\mathrm{Ca}: \mathrm{P}$ ratios are approximately 1.24 and 1.13 , respectively. These values are somewhat less than the stoichiometric $\mathrm{Ca}: \mathrm{P}$ ratio in HAP, which is 1.67 . 

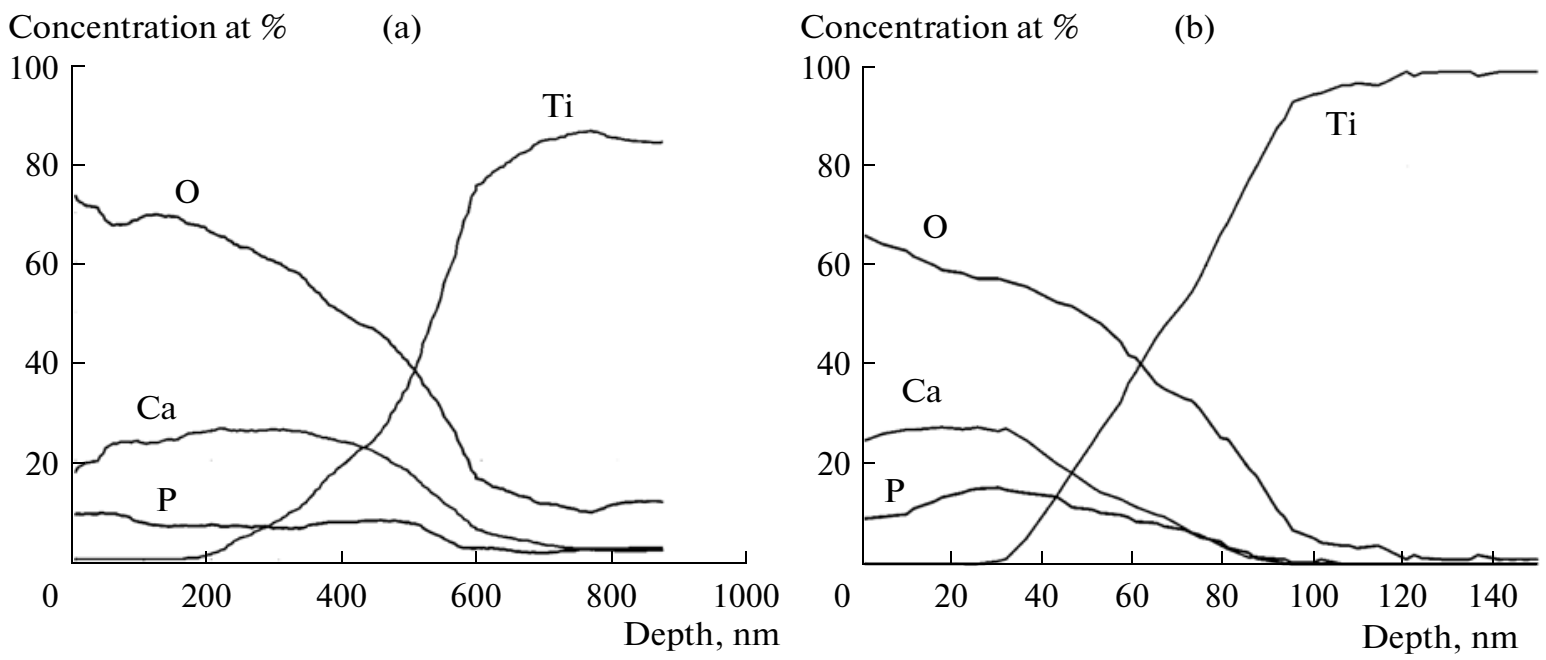

Fig. 2. Auger spectra of $\mathrm{CP}$ coatings deposited onto titanium substrates when (a) natural and (b) synthetic targets were irradiated by ion beams of a Temp-4M accelerator generating 300 current pulses.

In other experiments on the deposition of CP coatings under exposure to HPPIBs, the $\mathrm{Ca}: \mathrm{P}$ ratios were closer to the stoichiometric value $[4,5]$. This can be caused by a distinction between the HPPIB and target parameters.

The scanning electron microscopy (SEM) images, which were recorded during investigation of the surface morphology of CP coatings deposited on planar titanium substrates under the action of a series of 300 pulses, are presented in Fig. 3. The CP coatings obtained with the help of a synthetic target turn out to be thinner (Fig. 3b), repeat the morphology of the initial titanium substrate (Fig. 3a), and contain inclusions. It seems that these inclusions are target-material droplets. The surface of the coatings created by the erosion of a natural HAP target is modified to a larger extent (Fig. 3c). These coatings completely cover the substrate and fill initial scratches arisen from sample polishing.

The measured physical-mechanical properties (roughnesses, microhardnesses, and Young's moduli) are listed in Table 2. The CP coatings increase the initial substrate roughness and, consequently, the biotissue-implant contact area stimulating osteosynthesis

Table 1. Proportions of different elements in the CP coatings

\begin{tabular}{c|r|r|r|r}
\hline \multirow{2}{*}{ Element } & \multicolumn{2}{|c|}{ Synthetic HAP target } & \multicolumn{2}{c}{ Natural HAP target } \\
\cline { 2 - 5 } & wt \% & at \% & wt \% & at \% \\
\hline $\mathrm{C}$ & 12.41 & 25.24 & 14.90 & 28.79 \\
$\mathrm{O}$ & 27.85 & 42.54 & 28.97 & 42.02 \\
$\mathrm{P}$ & 4.35 & 3.43 & 5.39 & 4.04 \\
$\mathrm{Ca}$ & 5.41 & 3.30 & 6.09 & 3.52 \\
$\mathrm{Ti}$ & 49.97 & 25.49 & 44.65 & 21.63
\end{tabular}

and improving the bonding strength. The microhardnesses of the formed coating turned out to be higher than those of titanium and a cortical bone [12]. The elasticity moduli lie in the range of 6-14 GPa; i.e., they are comparable with the elasticity modulus of a cortical bone $(18 \mathrm{GPa})$. The foregoing confirms that products with such coatings are applicable as implants intended to replace bone tissues because they satisfy the requirements to bone implants.

The CP coating thicknesses measured via the profilometric method are depicted in Fig. 4. It is seen that the coating thickness increases linearly with increasing number of HPPIB pulses. For synthetic and natural targets, the coating-deposition rates (i.e., changes in its thickness per beam-current pulse) were, respectively, 0.65 and $4 \mathrm{~nm} /$ pulse at the given irradiation parameters. Thus, the natural HAP target erodes more intensely than the ceramic one fabricated from synthetic HAP. This circumstance is apparently related to the fact that the natural-target surface turned out to be more porous and rougher than the synthetic surface. This was due to the fact that the sintering temperature of the natural target was lower than that of the synthetic one (it is known that the lower sintering temperature makes it possible to create more porous materials with excess surface energy [13]). The developed surface relief must stimulate an increase in the rate of material removal from the surface due to evaporation.

The safety of the locally irritant action and hemolytic activity of medical products (materials) was ascertained via toxicological tests carried out at the certified test laboratory center of the Novosibirsk Research Institute of Hygiene. The tests results corroborate that metallic products with biocompatible $\mathrm{CP}$ coatings are untoxic and apyrogenic, do not initiate degenerative changes in tissue during implantation, and comply with the requirements of regulations. 


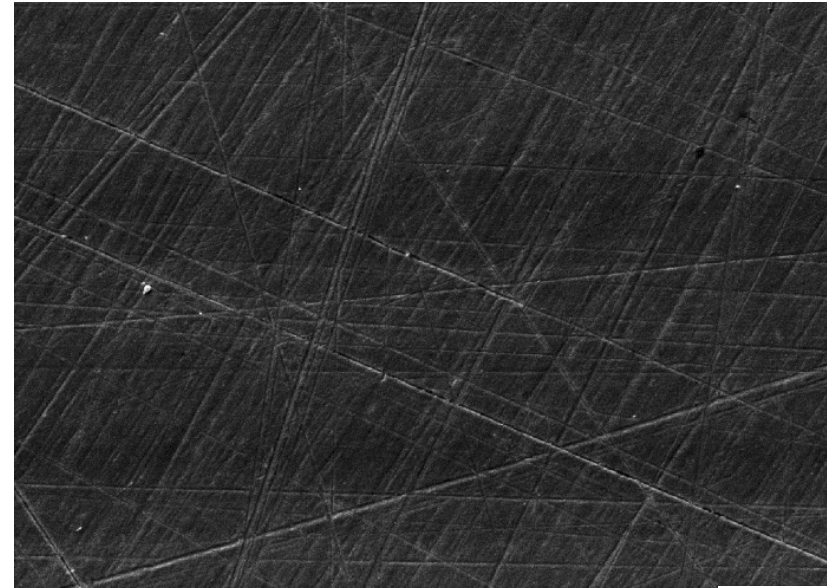

(a) $20 \mu \mathrm{m}$
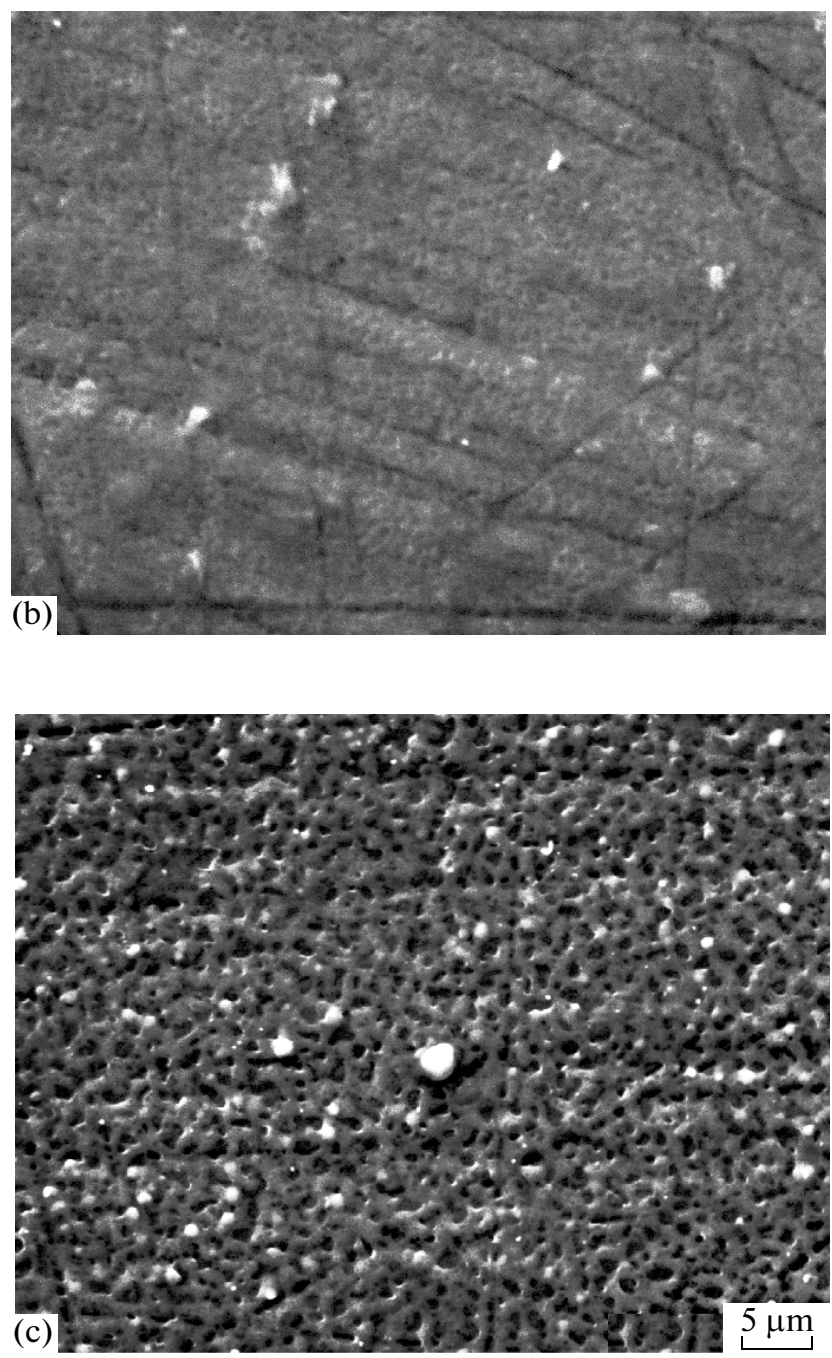

Fig. 3. SEM images of (a) the uncoated titanium substrate surface and CP coatings deposited onto titanium substrates under the irradiation of (b) synthetic and (c) natural targets (300 current pulses created by a Temp$4 \mathrm{M}$ accelerator).

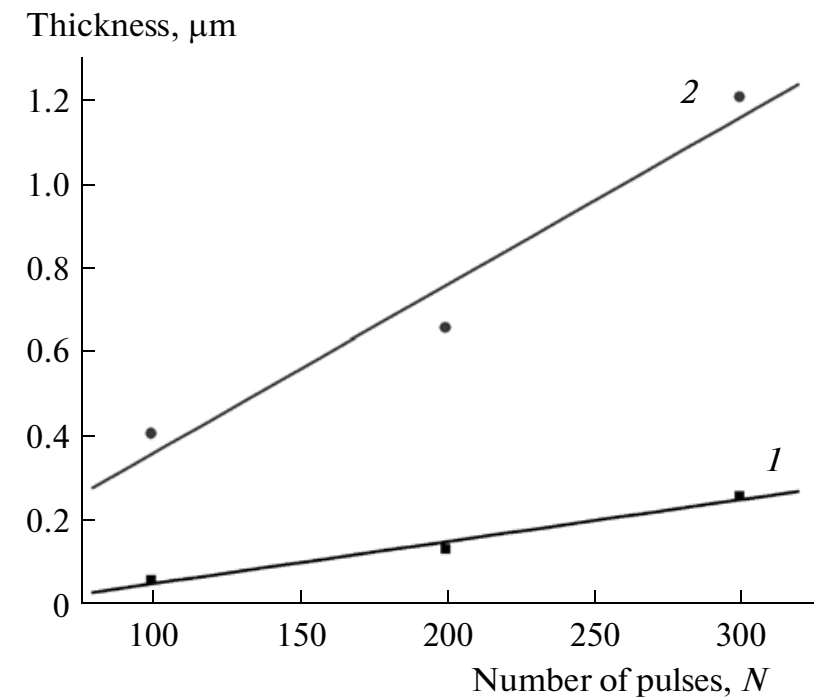

Fig. 4. Dependences between the CP coating thickness and the number of current pulses produced by a Temp-4M accelerator. The data were obtained for (1) synthetic and (2) natural targets.

\section{CALCULATION TECHNIQUE AND ANALYSIS OF RESULTS}

To understand the regularities of existing processes and predict deposition characteristics, knowledge of the mechanisms by which a substance is removed from target surface layers under the action of HPPIBs, i.e., erosion, is necessary. In $[8,9]$, it was shown that the solid surface erosion caused by incident HPPIBs with power densities of greater than $10^{7} \mathrm{~W} / \mathrm{cm}^{2}$ arises from the radiative heating of thin surface layers in the irradiated target. Substance liberation from the surface of the condensed phase occurs due to the transition into the vapor-plasma state. Let us consider a mathematical model of erosion under exposure to pulsed ion beams and electrons with initial energies of $10-1000 \mathrm{keV}$ and power densities of $10^{6}-10^{9} \mathrm{~W} / \mathrm{cm}^{2}$. It is assumed that its dominant mechanism is surface evaporation passing into hydrodynamic vapor expansion in the power range $P \geq 10^{9}-10^{10} \mathrm{~W} / \mathrm{cm}^{2}$. Owing to ion irradiation, an erosion flux must involve particles knocked out of the surface layers of a target during collisional sputtering. However, their fraction is less than $1 \%$ in the power range under study [8].

The intensity and energy efficiency of target-surface erosion and coating deposition are determined by the irradiation parameters. With the help of mathematical simulation, their dependences were previously found as applied to metallic materials $[8,9]$. In this paper, the calculation results were obtained for HAP targets irradiated by beams of mixed composition (different proportions of protons and carbon ions) with the initial particle energy $E_{0}=250-500 \mathrm{keV}$ and current-pulse durations from several tens of nanoseconds to several microseconds. The aforementioned HPPIB 
Table 2. Physical-mechanical properties of CP coatings

\begin{tabular}{l|c|c|c|c}
\hline \multicolumn{1}{c|}{ Substrate/Target } & $\begin{array}{c}\text { Number of HPPIB } \\
\text { pulses }\end{array}$ & $\begin{array}{c}R_{a}, \mu \mathrm{m} / \text { Roughness } \\
\text { class }\end{array}$ & $\begin{array}{c}\text { Elasticity modulus } \\
E, \mathrm{GPa}\end{array}$ & $\begin{array}{c}\text { Microhardness, } \\
H, \mathrm{GPa}\end{array}$ \\
\hline Ti/Synthetic HAP & 300 & $0.73 / 11 \mathrm{a}$ & $6.2 \pm 0.3$ & $3.5 \pm 0.4$ \\
\hline Ti/Natural HAP & 300 & $0.54 / 11 \mathrm{~b}$ & $14.5 \pm 1.3$ & $5.0 \pm 1.6$ \\
\hline \multicolumn{2}{c|}{$\mathrm{Ti}$} & $0.01 / 13 \mathrm{~b}$ & 110 & $\sim 1$ \\
\hline \multicolumn{2}{c|}{ Cortical bone } & - & $\sim 18$ & $0.51 \pm 0.01$ \\
\hline
\end{tabular}

parameters are inherent to modern ion accelerators capable of operating under industrial conditions. The energy efficiency of deposition was estimated according to the dependences between the coefficient of beam energy use in material evaporation from the target surface, $K_{\mathrm{ev}}$, and different irradiation parameters.

In the case of a HAP target, calculations based on the aforementioned model indicate that the behavior of $K_{\mathrm{ev}}$ vs. the HPPIB parameters is analogous to the dependence of metals: coefficient $K_{\mathrm{ev}}$ has a maximum appearing due to an increase in the current density $J$ and the energy density $F$, it increases with increasing initial beam particle energy $E_{0}$ and proton portion in it, and decreases with increasing current-pulse duration at a fixed beam energy density (Fig. 5). As is evident from Fig. 5, the value of $K_{\text {ev }}$ reaches $60 \%$ if the initial energy of the bombarding particles is several hundred kiloelectronvolts and submicrosecond current-pulse duration is used. An increase in the current-pulse duration from several tens of nanoseconds to several microseconds at constant fluence leads to an

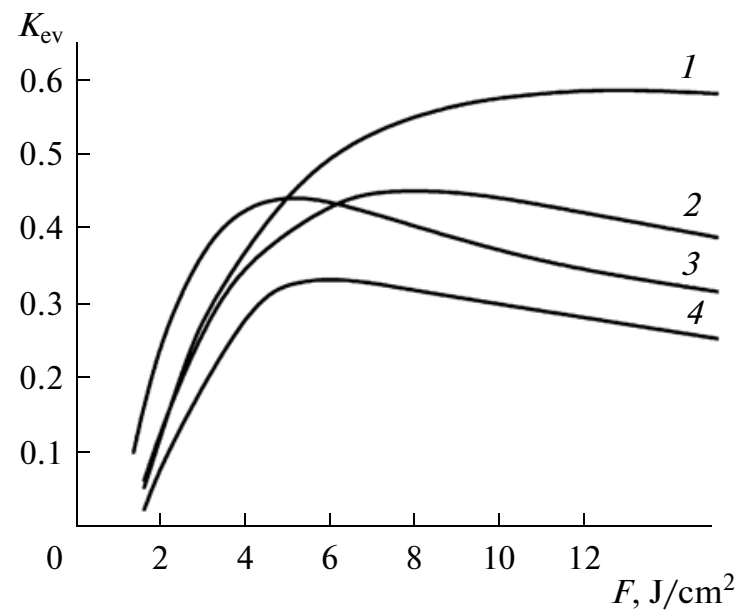

Fig. 5. Dependences between the beam-energy fraction expended on material evaporation from the HAP target surface, $K_{\mathrm{ev}}$, and the energy density of an ion beam of mixed composition: (1) $30 \%$ of $\mathrm{C}^{+}+70 \%$ of $\mathrm{H}^{+}, E_{0}=$ $250 \mathrm{keV}$, and $\tau=80 \mathrm{~ns}$; (2) $70 \% \mathrm{of} \mathrm{C}^{+}+30 \%$ of $^{+}, E_{0}=$ $500 \mathrm{keV}$, and $\tau=80 \mathrm{~ns}$; (3) $70 \%$ of $^{+}+30 \%$ of $\mathrm{H}^{+}, E_{0}=$ $250 \mathrm{keV}$, and $\tau=80 \mathrm{~ns}$; and (4) $70 \%$ of $\mathrm{C}^{+}+30 \% \mathrm{of}^{+}$, $E_{0}=250 \mathrm{keV}$, and $\tau=500 \mathrm{~ns}$. almost twofold decrease in the beam-energy fraction expended on the evaporation process.

Proton beams exhibit the largest energy deposition efficiencies. However, to achieve the maximum value of $K_{\mathrm{ev}}$, their energy density within the pulse must be much higher than that of beams consisting of heavier ions.

The coating growth rate $f_{\text {dep }}$ characterizing the efficiency of the technological process implemented by an accelerator operating in the pulse-frequency mode is defined as

$$
f_{\text {dep }}=Z_{\text {dep }} v
$$

where $Z_{\text {dep }}$ is the deposition rate or the coating-thickness increment within a single beam-current pulse and $v$ is the accelerator pulse frequency. The linear dependence of $f_{\text {dep }}$ on $Z_{\text {dep }}$ is confirmed by the results of experiments on coatings prepared from different materials, including CP coatings (Fig. 4), in modern accelerators.

Thus, when calculating the efficiency of the technology by which the coatings are deposited in the pulse-frequency mode of HPPIB irradiation, it is necessary to learn how to determine the coating-deposition rate.

Let us assume that coatings fabricated from erosion material created due to target irradiation by HPPIBs are deposited according to the diagram shown in Fig. 1. As a rule, the target and substrate surfaces are parallel or are located at a small angle to each other. The beam cross-section area is usually several tens of square centimeters. The target area is less than or equal to the beam cross-section area, thereby making it possible to avoid the unwanted sputtering of structural elements.

Let erosion material be generated on the target surface with the known rate $V$ at any instant $t$. This rate can be calculated, e.g., with the help of the aforementioned surface evaporation model [8]. It is assumed that the material evaporated from the unit area $i$ on the target surface is emitted within spatial angle $2 \pi$ and the flux density $I$ of evaporated atoms (molecules) has cosine distribution over the departure angle $\varphi$ to the normal to the target surface:

$$
I(\varphi)=I_{0} \cos \varphi
$$


where $I_{0}$ is the material flux density in the direction perpendicular to the target surface.

Using the second Lambert-Knudsen law [14], we obtain the following formula for calculating the rate at which the thickness of the coating deposited from the material evaporated from the unit area $i$ on the target surface $d S$ grows at the instant $t$ :

$$
V_{\mathrm{dep} i}=\frac{v d S \cos ^{4} \varphi_{i}}{\pi L^{2}}
$$

Here, $L$ is the distance between the target and substrate surfaces and $\varphi_{i}$ is the angle between the normal to the surface of the unit area $i$ on the target and the material deposition direction. Therefore, with allowance for material removal from the entire evaporation region on the target surface with the area $S_{C}$, the coating thickness $Z_{\text {dep }}$ at the substrate point with coordinates $(x, y)$, which is deposited within a single current pulse, can be calculated from the formula

$$
=\frac{1}{\rho_{f}} \int_{0}^{t_{\mathrm{end}}} \frac{\mathrm{v}(t)}{\pi} \iint_{S_{c}} \frac{Z_{\mathrm{dep}}(x, y)}{\left(L^{2}+\left(x-x_{c}\right)^{2}+\left(y-y_{c}\right)^{2}\right)^{2}} d t,
$$

Here, $t_{\text {end }}$ is the estimated time of evaporation cessation, which is counted from the onset of the interaction between the current pulse and the target; $\rho_{f}$ is the deposited film density; and $V$ is the instantaneous evaporation rate at the instant $t$.

The deposition rate and the intensity and energy efficiency of the material removal from the HAP target surface vs. different beam parameters were determined. The parameters were selected on the basis of the potential of modern pulsed ion accelerators developed to satisfy technological purposes.

Figure 6 illustrates the dependences between the $\mathrm{CP}$ coating deposition rate and the current density $J$ of the ion beam of the Temp-4M pulsed accelerator. The maximum coating thickness is hereinafter assumed to be attained at the substrate-surface point facing the target center. The continuous curves designate data calculated according to the technique described above, and the dots correspond to experimental results obtained for the case of analogous irradiation parameters. Good agreement between the calculated and measured data implies that model approximations to the erosion mechanism and the deposition-rate-calculation technique are suitable for predicting the CP coating deposition efficiency if coatings are formed by means of HPPIBs.

With the use of such beams, the current density threshold ensuring the formation of $\mathrm{CP}$ coatings varies from 100 to $300 \mathrm{~A} / \mathrm{cm}^{2}$, as is dictated by the initial particle energy and the beam composition. When the pulse frequency is $0.1-1.0 \mathrm{~Hz}$, i.e., corresponds to the

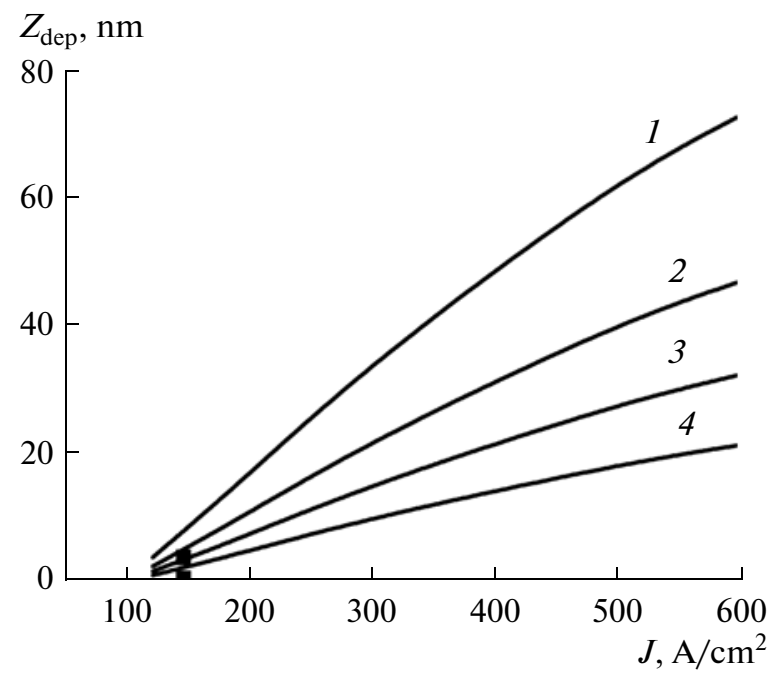

Fig. 6. Dependences between the CP coating deposition rate, $Z_{\mathrm{dep}}$, and the current density of an ion beam of mixed composition $\left(70 \%\right.$ of $\mathrm{C}^{+}+30 \%$ of $\left.\mathrm{H}^{+}\right)$. The initial particle energy is $250 \mathrm{keV}$, the pulse duration is $80 \mathrm{~ns}$, and the distances between the target and the substrate are (1) 5 , (2) 6.5 , (3) 8 , and (4) $10 \mathrm{~cm}$. The continuous curves correspond to calculation data and the dots are experimental data obtained by the irradiation of targets fabricated from (upper dot) natural and (lower dot) synthetic HAP.

capabilities of modern pulse-frequency accelerators, the CP coating deposition efficiency is $0.1-100 \mathrm{~nm} / \mathrm{s}$.

Figure 7 presents the dependences between the CP coating deposition rate and the energy density $F$ of an ion beam having mixed composition with different portions of protons and carbon ions and different initial-particle energies $E_{0}$. When quantity $F$ and the beam composition have identical values, an insignificant distinction in the deposition rates is observed at different values of $E_{0}$. At a low level of $F$, the quantity $Z_{\text {dep }}$ is higher at smaller values of $E_{0}$. An increase in the energy density changes the situation: the higher the initial beam particle energy, the higher the deposition rate. The beam composition also affects the $Z_{\text {dep }}(F)$ behavior: the higher the number of protons in the beam, the higher the deposition rate at identical beam energy densities. When the current-pulse duration becomes longer at constant fluence, the deposition rate slightly decreases because the beam energy portion transferred from the particle deceleration region to the bulk of the target increases due to heat conduction.

It follows from Fig. 7 that all dependences $Z_{\text {dep }}(F)$ are characterized by a rapid increase in deposition rate with increasing $F$ at low beam-energy densities and, thereafter, reach saturation with increasing $F$. This mechanism is related to the fact that the beam energy loss increases in the vapor environment and, consequently, erosion material is generated less intensively. 


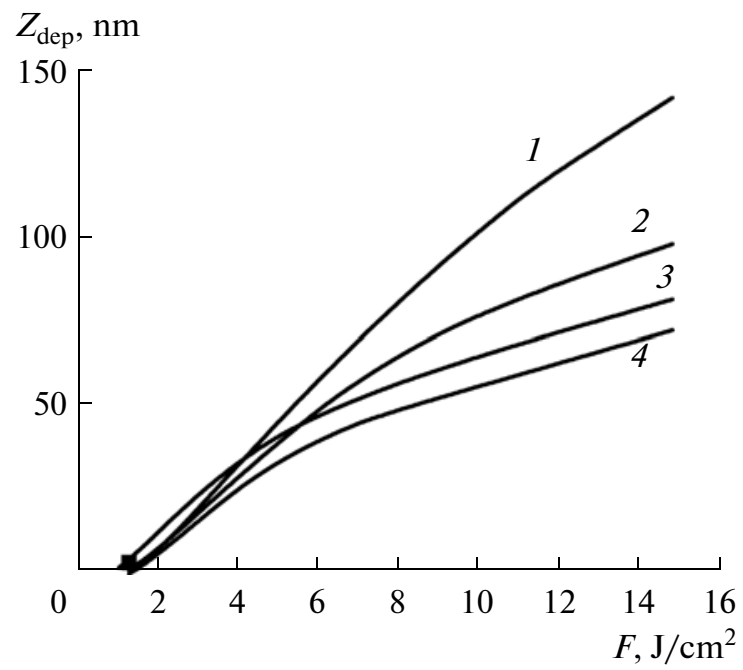

Fig. 7. Dependences between the CP coating deposition rate and the energy density of an ion beam with mixed composition, different pulse durations $\tau$, and different initial particle energies $E_{0}$. The beam is incident on the target at an angle of $45^{\circ}$ to the normal to its surface, the beamtrace radius is $2.5 \mathrm{~cm}$, and the distance between the target and the substrate that are parallel to each other is $6.5 \mathrm{~cm}$. Here, (1) $30 \%$ of $\mathrm{C}^{+}+70 \%$ of $\mathrm{H}^{+}, E_{0}=250 \mathrm{keV}$, and $\tau=$ $80 \mathrm{~ns}$; (2) $70 \%$ of $\mathrm{C}^{+}+30 \%$ of $\mathrm{H}^{+}, E_{0}=500 \mathrm{keV}$, and $\tau=$ $80 \mathrm{~ns}$; (3) $70 \%$ of $\mathrm{C}^{+}+30 \%$ of $\mathrm{H}^{+}, E_{0}=250 \mathrm{keV}$, and $\tau=80 \mathrm{~ns}$; and (4) $70 \%$ of $\mathrm{C}^{+}+30 \%$ of $\mathrm{H}^{+}, E_{0}=250 \mathrm{keV}$, and $\tau=500 \mathrm{~ns}$.

\section{CONCLUSIONS}

Analysis of the capabilities offered by modern submicrosecond ion accelerators of the Temp-4M type indicates that, in principle, they can be used to deposit calcium phosphate coatings on the metallic base of new-generation bone implants. The formed CP coatings contain dsirable chemical elements and satisfy biomedical requirements to the microhardness and the level of Young's modulus. They completely cover the substrate surface and exhibit good adhesion to the base material. The technology of fabrication of hydroxyapatite targets affects the properties and deposition rate of $\mathrm{CP}$ coatings. Porous and rough targets are capable of ensuring a higher coating formation rate. Good agreement between experimental results and calculated CP coating deposition rates confirms that the basic mechanism behind the generation of the deposited material is atomic and molecular evaporation from the surface layers of an irradiated target. The dependences of the productivity and energy efficiency of deposition on the beam parameters have been determined via numerical simulation. Calculations enable us to infer that the beam parameters used in the experiments are not optimal. To improve the productivity and energy efficiency of the technology, the generation of beams with a predominant content of pro- tons and energy densities of $5-10 \mathrm{~J} / \mathrm{cm}^{2}$ are advisable (under the condition that deposited coatings will meet biomedical requirements).

\section{ACKNOWLEDGMENTS}

This study was performed under the Nauka state task and supported in part by the Research and Development in Priority Fields of Russian Scientific and Technological Complex for 2007-2013 federal target program (state contract no. 14.512.11.0012), the Russian Foundation for Basic Research (project no. 1108-98032-p_sibir' a), and Research Government Task no. $0.3 \overline{2} 8.201 \overline{2}$ (project no.7.1084.2011).

\section{REFERENCES}

1. I. F. Isakov, G. E. Remnev, and A. N. Zakutaev, in Proceedings of the 9th International Conference on HighPower Particle Beams (NTIS, Springfield, 1992), p. 1966.

2. T. J. Renk, P. P. Provencio, S. V. Prasad, et al., Proc. IEEE 92, 1057 (2004).

3. K. Yatsui, T. Sonegawa, K. Ohtomo, and W. Jiang, Mater. Chem. Phys. 54, 219 (1998).

4. V. K. Struts, A. V. Petrov, V. M. Matvienko, et al., J. Surf. Invest.: X-ray, Synchrotron Neutron Tech. 5, 497 (2011).

5. S. I. Tverdokhlebov, G. E. Remnev, A. I. Pushkarev, et al., in Proceedings of the Scientific-Practical Conference on Modern Ceramic Materials and their Application (Sibprint, Novosibirsk, 2010), p. 111.

6. M. S. Saltymakov, S. I. Tverdokhlebov, A. I. Pushkarev, and T. L. Volokitina, in Proceedings of the 18th International Conference on High-Power Particle Beams (ICC Jeju Jeju, Korea, 2010), p. 236.

7. V. F. Pichugin, E. V. Eshenko, R. A. Surmenev, et al., J. Surf. Invest.: X-ray, Synchrotron Neutron Tech. 1, 679 (2007).

8. G. A. Bleykher and V. P. Krivobokov, J. Eng. Thermophys. 17, 24 (2008).

9. G. A. Bleikher and V. P. Krivobokov, Izv. Vyssh. Uchebn. Zaved., Poroshk. Metallurg., No. 1, 23 (2008).

10. Yu. I. Isakova, A. I. Pushkarev, and V. A. Tarbokov, Izv. Tomsk. Politekh. Univ. 316 (2), 76 (2010).

11. M. V. Chaikina, Mechanochemistry of Natural and Synthetic Apatites (Sib. Otd. RAN, Novosibirsk, 2002) [in Russian].

12. P. Li, PhD Thesis (Leiden, 1993).

13. K. K. Strelov, Theoretical Principles of the Technology of Refractory Materials (Metallurgiya, Moscow, 1985) [in Russian].

14. HandBook of Thin Film Technology, Ed. by L. Meisel and R. Glang (McGraw-Hill, New York, 1970; Sov. radio, Moscow, 1977), Vol. 1.

Translated by S. Rodikov 